\title{
Identidad Narrativa en cuidadoras informales familiares y no familiares de personas en diálisis
}

\author{
Identity Narrative in informal family and non-family \\ caregivers of people on dialysis
}

\author{
Luisa Seco-Lozano \\ Hospital General Universitario de Valencia, Valencia, España. \\ $\bowtie$ luisec31@yahoo.es \\ (D) https://orcid.org/0000-0002-0773-3413
}

\section{Sacramento Pinazo-Hernandis}

Universidad de Valencia, Valencia, España.

$\triangle$ sacramento.pinazo@uv.es

(i) https://orcid.org/0000-0003-4364-4730

Agustín Domingo-Moratalla

Universidad de Valencia, Valencia, España

$\triangle$ agustin.domingo@uv.es

https://orcid.org/0000-0001-8848-3761

Recibido: 04-04-2019

Aceptado: 08-06-2019

Publicado: 12-06-2019

\section{RESUMEN}

Objetivo. Explorar la identidad narrativa de las cuidadoras informales familiares o no, de personas en diálisis. Método. Entrevistas en profundidad a 9 cuidadoras de personas de programas de Hemodiálisis o Diálisis Peritoneal del Hospital General Universitario. Se obtuvo autorización del Comité de Ética y los consentimientos informados de los participantes. El análisis se hizo desde la Teoría Fundamentada. Las narraciones transcritas y codificadas fueron sometidas a reflexión hermenéutica. Resultados. La enfermedad genera un impacto y las cuidadoras asumen el cuidado como pueden. Surge el sentimiento de "cuidado como destino" y como dotación intrínseca de "ser mujer", percibido como una especie de fatalismo o de inexorabilidad. Conclusiones. Existe un desconocimiento acerca de algunas tareas del cuidado informal tan relevantes como: el acompañamiento, la escucha o la convivencia, que permanecen totalmente invisibles para la sociedad, así como una falta de arbitrajes entre las relaciones cuidadoras informales familiares y no familiares. Por otra parte, la relación de cuidado no puede construirse sin dos ingredientes fundamentales: el cariño y la confianza. Estas cualidades son vinculantes y recíprocas en el cuidado informal en el contexto de una relación de familiaridad. Cuidar es convivir y la vida de las cuidadoras informales de las personas en diálisis crónica es, en muchas ocasiones, vivir cuidando.

Palabras clave: Cuidado informal; identidad narrativa; diálisis crónica; teoría fundamentada; hermenéutica. 


\section{ABSTRACT}

Objective. To explore the narrative identity of informal caregivers, family or nonfamily, of people on dialysis. Method. In-depth interviews with 9 caregivers of people in Hemodialysis or Peritoneal Dialysis programs at the University General Hospital. Authorization was obtained from the Ethics Committee and the informed consent of the participants. The analysis was made from the Grounded Theory. The transcribed and coded narrations were subjected to hermeneutic reflection. Results. The disease has an impact and the caregivers take care of it as best they can. The feeling of "care as destiny" and as an intrinsic endowment of "being a woman" arises, perceived as a kind of fatalism or inexorability. Conclusions. There is a lack of knowledge about some informal care tasks as relevant as: accompaniment, listening or coexistence, which remain totally invisible to society, as well as a lack of arbitrage between informal family and non-family caregiving relationships. On the other hand, the caring relationship cannot be built without two fundamental ingredients: affection and trust. These qualities are binding and reciprocal in informal care in the context of a family relationship. Caring is living together, and the life of informal caregivers of people on chronic dialysis is often living in care.

Keywords: Informal care, narrative identity. Chronic dialysis, Grounded Theory, Hermeneutics.

\section{INTRODUCCIÓN}

Cuidar a una persona con dependencia supone un gran cambio en la vida de la persona que va a cuidar. Durante el primer año de cuidado es cuando se produce el mayor número de cambios. Los motivos que llevan a las personas cuidadoras familiares a ejercer este rol son muy diversos: cariño y obligación filial y conyugal se superponen como los más destacados; la presión social, la gratificación, el rechazo a la institucionalización, el respeto a la opinión del dependiente o la disponibilidad del momento también están presentes (Moral, Frías, Moreno, Palomino y del Pino, 2017).

Las cuidadoras informales presentan unas características bastante similares. Se trata de mujeres, con una edad intermedia entre 50 y 80 años, no tienen empleo y si lo tuvieron han tenido que abandonarlo, lo que les conlleva dificultades económicas. Dedican más de 40 horas semanales al cuidado, asumiendo casi en exclusiva el cuidado de la persona dependiente por lo que tienen dificultad para mantener sus relaciones sociales, no tienen tiempo para cuidarse y padecen de patologías múltiples: cansancio, dolores de espalda, problemas de sueño y frecuentemente depresión (Durán, 2018).

El género impregna la totalidad de estructuras de cuidados (...). Por su papel en la reproducción de la vida se atribuye a las mujeres determinadas capacidades y habilidades para cuidar y esto contribuye a que estén fuertemente naturalizadas. Queda así oculto que el aprendizaje es un componente básico en estas actividades (Comars, 2015). Es importante remarcar que la decisión de cuidar no se toma de forma individual, sino que es una decisión colectiva, familiar. El género y la posición familiar son determinantes en la configuración de la co-responsabilidad del cuidado informal (Rogero, 2009). 
Seco-Lozano et al - Identidad Narrativa en cuidadoras informales familiares y no familiares de personas en diálisis.

El cuidado informal también presenta unas consecuencias sobre la salud del cuidador que impactan de forma negativa en éste. Las relacionadas con la salud son de carácter emocional: estrés psicológico, pérdida de control y autonomía. El impacto económico y en las relaciones sociales también son aspectos relevantes (Rogero, 2009). Pero también hay estudios que intentan profundizar en los aspectos más positivos del cuidado. Las cuidadoras que encuentran sentido en el cuidado familiar tienen mayor bienestar, menor sobrecarga y afrontan mejor las tareas del cuidado (Farran, 2010, citado por Martínez y Benjumea 2015, p. 82). Continuando con la argumentación de la autora: "Esta idea, de que es la familia quien se responsabiliza del cuidado oculta que los cuidados conciernen al conjunto de la sociedad ya que afectan a la reproducción social. Es necesario remarcar el modelo familista de cuidado no favorece la visibilidad de esta realidad, ya que en su discurso es la familia la proveedora del cuidado en general, sin embargo, en muchas ocasiones se obvia decir que el cuidado lo provee la mujer sin recibir remuneración, reconocimiento ni apoyo ya que su rol de proveer cuidado está instalado en la normativa social.

Los cuidados que precisan las personas en diálisis no son siempre iguales, ya que cada situación es única. Serán las características personales, y el contexto en el que se desarrolle la enfermedad las que marcarán la pérdida de autonomía. Gran parte del cuidado que necesitarán lo aportarán las cuidadoras familiares (Brassoloto y Daly, 2016).

El impacto emocional que la enfermedad renal y su tratamiento tiene sobre estas personas es muy grande. Se han descrito tres tipos de impacto: el primero, cuando al interactuar con los proveedores principales de cuidado sienten que su tratamiento es una carga 0 una rutina, esto les lleva a sentir experiencias de abandono, soledad o alienación; el segundo tipo, cuando al enfrentarse a la organización sanitaria surgen sentimientos similares a los ya descritos; y el tercer impacto es el tratar de construir un sentido a la nueva vida ya que debido a su enfermedad en muchas ocasiones se culpabilizan a sí mismos pensando que pudo haberse prevenido (O'Hare et al., 2018).

Las cuidadoras de personas en diálisis proporcionan ayuda cuidados instrumentales, ayudan en el autocuidado y acompañan durante toda la enfermedad, que representa un período de tiempo ilimitado. Estas cuidadoras presentan unas necesidades y demandas muy altas de información, apoyo económico y apoyo emocional (Ramos y Jarillo, 2014).

Estas cuidadoras tienen unas características; suelen compartir de forma mayoritaria el mismo hogar que la persona a la que cuidan y el cuidado lo hacen en solitario y a tiempo completo. Suelen presentar ansiedad en niveles medios y altos, y aparece con frecuencia la depresión (Romero y MacCausland y Solórzano, 2014). No pueden disponer de tiempo libre lo que afecta a las relaciones sociales y laborales, con la consecuente afectación del nivel económico y relacional (Zamarripa, Támez y Ribeiro, 2017).

Por otra parte, para el $70 \%$ de los hogares españoles es imposible pagar a un cuidador a jornada completa, ya que consumiría más de un tercio de los ingresos del hogar. Ante esta situación, aunque las cuidadoras familiares cuenten con sus propios recursos de tiempo de cuidado no remunerado, a menudo es insuficiente (Durán, 2018).

En el cuidado informal no sólo participan los familiares, sino que hay todo un mercado que sustituye esta atención familiar: las cuidadoras informales no familiares. El perfil de 
estas cuidadoras sigue siendo el de una mujer, pero esta vez inmigrante con necesidad de trabajar, pudiendo tener o no tener formación previa en el mundo del cuidado.

Encontramos entonces que el contexto en el que se realiza el trabajo de cuidado se genera en la interconexión de los regímenes de cuidados existentes y la situación migratoria. Podemos ver como la disponibilidad del recurso de cuidadoras informales a un coste asequible por las familias, así como los recursos institucionales y la propia cultura delimitan las posibilidades de cuidado en una sociedad (Moré, 2016).

Estudiar el cuidado informal en el contexto de la cronicidad de las personas en diálisis puede ayudar a determinar las implicaciones sociales y en salud que tiene el cuidado en una sociedad cada vez más envejecida, especialmente la población de personas mayores en tratamiento crónico en diálisis.

\section{MÉTODO}

Se ha utilizado un enfoque narrativo, entendiendo que la identidad narrativa es aquella que el sujeto alcanza por mediación de la función narrativa (Ricoeur, 1999). Esta idea expresa que la experiencia humana se caracteriza por ser narración, se estructura narrativamente, y lo racional está mezclado con esa narratividad, es decir, con el contexto, lo subjetivo, aquello vital que experimento solo yo y que comunico desde mi percepción única. Como expresa Domingo (2007) "Las narraciones forman parte de nuestra vida. Somos narradores de nosotros mismos. También las fuentes morales, nuestras convicciones, nuestras creencias, son narrativas. La identidad humana, personal, social e histórica es narrativa" (p. 285).

La narración introduce el sentido y la realidad del otro, pero además permite introducir la dimensión temporal de la persona, encontrándonos con toda la complejidad que eso conlleva: el holismo, la integralidad. Es decir, no tratamos con identidades inmutables sino con personas donde el todo emerge en cada una de sus partes: lo biológico, lo social, lo psicológico (Parra, 2014).

La identidad narrativa aparece así como un recurso teórico de gran potencial para explicar situaciones de cambio o crisis vitales en donde se ponen en juego los cambios de significados o refiguraciones y sus resoluciones o configuraciones (Iacub, 2013).

Diseño del estudio. Se definió el problema de investigación con la pregunta inicial: ¿Cómo viven la experiencia del cuidado las cuidadoras informales familiares y no familiares de las personas en diálisis?

Se diseñó un abordaje que combina el análisis de texto, codificación y categorización de la Teoría Fundamentada junto a una reflexión hermenéutica de las narraciones con el propósito de contextualizar el fenómeno del cuidado informal en las personas en diálisis.

Para ello se diseñó una entrevista, como instrumento para la recogida de datos ,que consta de 10 áreas y 41 preguntas abiertas que la persona puede contestar con libertad ,e incluso hablar de otros temas que considere necesarios para aclarar sus puntos de vista. Seguidamente se enumeran las 10 áreas: 
Seco-Lozano et al - Identidad Narrativa en cuidadoras informales familiares y no familiares de personas en diálisis.

1. Compromiso en el cuidado

2. $\quad$ Significado del cuidado /autocuidado

3. Responsabilidades de cuidado/autocuidado

4. $\quad$ Cultura y Tradición en el cuidado y autocuidado

5. $\quad$ Entornos: rural, urbano, redes sociales

6. Aprendizaje cuidado/autocuidado

7. Tareas que exige el cuidado

8. Instituciones y cuidado

9. Relaciones personales y cuidado

10. Aspectos técnicos de la hemodiálisis y diálisis peritoneal

Análisis. Para el análisis narrativo se utilizan dos enfoques cualitativos: un análisis estructural, donde se analiza el texto de forma exhaustiva con las herramientas de la Teoría Fundamentada (Método Strauss-Corbin) y un análisis hermenéutico que conceptualiza la temática que surge fuera de las áreas que se habían delimitado con anterioridad.

Análisis de datos. Las estrategias de obtención y análisis de datos de la Teoría Fundamentada que utilizamos son: codificación, muestreo teórico y comparación constante, con el objetivo de llegar a la saturación teórica de los datos. Para ello se ha tratado el texto a analizar de la siguiente forma. Las entrevistas fueron grabadas y posteriormente transcritas de forma cronológica, es decir, no se realizaría la siguiente entrevista hasta que se contara con la transcripción de la anterior. En esa transcripción también se incluían las anotaciones del diario de campo.

Codificación abierta: análisis "línea por línea", análisis "oración o párrafo", examen del documento entero y formulación de preguntas.

Codificación axial: categoría, propiedad de la categoría, hipótesis.

Para la reflexión hermenéutica se abordaron las narraciones desde la perspectiva hermenéutica de Paul Ricoeur, donde la interpretación de los fenómenos particulares se realiza desde un paradigma humanista con un énfasis especial en el contexto/tiempo en que ocurren. Desde este enfoque se analizaron los temas /categorías que emergieron de forma espontánea en las narraciones y que no se habían categorizado desde la Teoría Fundamentada.

Las medidas para garantizar la validez interna y externa del estudio que se llevaron a cabo fueron realizar una comprobación de coherencia por dos profesionales ajenos al estudio y una triangulación por datos.

Descripción de la muestra. La muestra la conforman 9 mujeres en total, 3 de ellas son cuidadoras no familiares y 6 son cuidadoras familiares de las personas en diálisis. De las tres mujeres cuidadoras no familiares sólo una de ellas es española, las otras dos son de origen latino. Las cuidadoras familiares son hijas (3) o esposas (3) de las personas cuidadas y llevan años (10-5 años) ejerciendo su rol.

La mayoría de las mujeres entrevistadas trabajan y compaginan ese trabajo con el cuidado, excepto una de ellas que se encuentra jubilada. A pesar de realizar un trabajo fuera de casa, la dedicación en tiempo al cuidado para ellas es importante. La mujer de 
más edad que está jubilada se dedica por completo al cuidado de su marido. Sólo dos de ellas cuentan con apoyos efectivos para cuidar. En un caso se trata de una hija joven, que aún está estudiando y que todavía convive en el domicilio, y en otro caso se trata de un cuidado compartido entre varias hermanas. Las demás mujeres cuidan en solitario y recurren o han recurrido al cuidado remunerado de una cuidadora informal. Tienen un nivel de estudios grado medio y su residencia es en Valencia capital o cercanías, excepto una persona que vive en una zona rural bastante alejada de la ciudad. Estas mujeres tienen muy restringido el ocio mayoritariamente, y presentan problemas de salud que se ven agravados al no disponer de tiempo para su propio autocuidado.

Diseño muestral. Se seleccionó la muestra teniendo en cuenta la relevancia que tiene para el estudio que sean cuidadoras de personas en diálisis crónica y no de otra enfermedad o contexto. La muestra se obtuvo por la técnica de bola de nieve al iniciar las dos primeras entrevistas a cuidadoras familiares de personas en diálisis del Hospital General Universitario de Valencia. La muestra fue universal, alcanzando la saturación de datos. Las variables que se buscaban fueron: cuidar a una persona en diálisis crónica durante más de seis meses.

Tabla 1. Datos sociodemográficos

\begin{tabular}{|c|c|c|}
\hline DATOS & CINF(N3) & $\operatorname{CIF(6)}$ \\
\hline Edad & MEDIA (DT=38-42) & MEDIA (DT=52-68) \\
\hline Género & $100 \%$ Mujeres & $100 \%$ Mujeres \\
\hline Tiempo que lleva cuidando & MEDIA (DT:10-5años) & MEDIA (DT=1-15 años) \\
\hline Situación laboral & $100 \%$ Activas & $90 \%$ activas $10 \%$ Jubilada \\
\hline Estado civil & $\begin{array}{l}90 \% \text { Casadas } \\
10 \% \text { Solteras }\end{array}$ & $\begin{array}{c}60 \% \text { Casados } \\
20 \% \text { Solteras } \\
20 \% \text { Separados }\end{array}$ \\
\hline Nivel de estudios & $\begin{array}{c}90 \% \text { Primaria } \\
10 \% \text { Universitarios }\end{array}$ & $\begin{array}{l}30 \% \text { Sin estudios } \\
50 \% \text { Grado medio (FP) } \\
20 \% \text { Universitarios }\end{array}$ \\
\hline Zona residencia & $100 \%$ Urbana & $90 \%$ Urbana \\
\hline Pueblo/localidad & $100 \%$ Valencia & $80 \%$ Valencia \\
\hline ¿Vive solo? & $100 \%$ No & $100 \%$ No \\
\hline ¿Conoce y/o utiliza las nuevas tecnologías? & $100 \% \mathrm{Si}$ & $80 \% \mathrm{Si}$ \\
\hline ¿Cómo es su estado de salud? & $\begin{array}{c}90 \% \text { Bastante bueno (Soy fuerte) } \\
10 \% \text { Regular }\end{array}$ & $\begin{array}{c}\text { 70\%Simplemente bueno } \\
20 \% \text { Regular } \\
10 \% \text { Precario (he perdido mucho) }\end{array}$ \\
\hline ¿Padece alguna enfermedad o problema de salud? & Asma y HTA (1) & $\begin{array}{c}\text { Espalda, osteoporosis (2) } \\
\text { Ansiedad (1) } \\
\text { Colesterol (1) }\end{array}$ \\
\hline Dedica tiempo a su cuidado. Revisiones médicas & $100 \% \mathrm{Si}$ & 60\% Sí \\
\hline Dieta adecuada & 100\% Sí & 60\% Sí \\
\hline Higiene y aseo personal & $100 \% \mathrm{Si}$ & 100\% Sí \\
\hline Descanso/sueño & 100\% Sí & 80\% Sí \\
\hline Medidas rehabilitadoras & 50\% Sí & $100 \%$ No \\
\hline Ejercicio físico adecuado & 90\% Sí & $80 \%$ No \\
\hline Toma de medicación adecuada & 100\% Sí & 100\% Sí \\
\hline Ocio y actividades recreativas & 100\% Sí & $80 \%$ No \\
\hline Relaciones afectivas y sociales & 100\% Sí & $10 \%$ No \\
\hline
\end{tabular}


Seco-Lozano et al - Identidad Narrativa en cuidadoras informales familiares y no familiares de personas en diálisis.

\section{RESULTADOS}

La identidad narrativa de cuidadoras familiares y no familiares y los significados de cuidado se entrelazan en los relatos, formando parte de esa identidad las formas de conceptualizar el cuidado. Se han encontrado 8 categorías y 3 temas que surgen de las narraciones y que se describen a continuación:

Tabla 2. Descripción de categorías

\begin{tabular}{cc}
\hline \multicolumn{2}{c}{ Categorías narrativas cuidadoras } \\
\hline $\begin{array}{c}\text { 1-Obligaciones del afecto y expresión del cuidado } \\
\text { 2-Cuidar como destino }\end{array}$ & $\begin{array}{c}\text { 5-Cuidar y convivir } \\
\text { 6-El olvido del cuidado } \\
\text { 3-¿as familias hacen lo que pueden } \\
\text { 7-La tarea de acompañar }\end{array}$ \\
\hline \multicolumn{2}{c}{ Temas análisis hermenéutico } \\
1-Vivir para cuidar & 3-Tensiones en el cuidado informal \\
\hline
\end{tabular}

\section{Categorías narrativas teoría fundamentada}

Categoría 1: Obligaciones del afecto y expresión del cuidado. Las cuidadoras familiares se sienten comprometidas a cuidar de sus familiares, especialmente ascendientes, descendientes y pareja. El vínculo del afecto proporciona el cuidado, y a la vez el cuidado es expresión de ese afecto. Es un afecto activo, práctico y decidido a la acción. Es un compromiso vivo y actualizado en el que el cuidado actúa de unión, es elemento catalizador del afecto. En ocasiones este "afecto cuidadoso" se siente de manera más expandida hacia el entorno cercano y también se siente atraído por la vulnerabilidad y por aspectos sociales y emocionales:

"Me siento comprometida a cuidar cuando surgen dificultades laborales, o hay desestabilidad emocional, y claro de mis nietas, mis hijas y mi marido." (CF4)

Esta categoría se define por la necesidad de vehiculizar el afecto mediante el cuidado. Este afecto que une establece lazos y compromisos es el que dota de significados al cuidado. Sin el vínculo afectivo, el cuidado no se expresa en un "buen cuidado". El afecto conlleva obligaciones, que aquí se traducen en cuidado, y ese cuidado a su vez se traduce en cualidades para que al ser ejercido se convierta en un buen cuidado. En ocasiones, cuando la capacidad de cuidar está sobrecargada, o cuando no se desea cuidar, el cuidado vehiculiza el malestar, la culpa, la exigencia, el sacrificio que supone el dar, el dedicar, el decidir estar en disposición de ayudar.

"Cuidar es una dedicación de parte de tu vida, de tu tiempo cuidando." (CF7)

"Cuidar de alguien es una obligación que no tienes más remedio cuando quieres a la persona." (CF3)

Es una obligación moral que nace del cariño y que abarca a la persona de forma integral.

"Cuidar de alguien es estar dispuesto a prestarle ayuda... pues en lo que no pueda realizar por sí mismo: puede ser algo físico, puede ser acompañarle, estar con él, hacerle compañía y que se sienta escuchado." (CF6) 
Para las cuidadoras no familiares los significados del cuidado son un trabajo, pero también es algo más, porque a veces un trabajo puede hacerse sin ganas, con mejor o peor humor, pero el trabajo del cuidado siempre requiere del ingrediente del cariño, la empatía y la paciencia:

"Es estar pendiente de las necesidades de esa persona, no solo a nivel físico, sino a nivel emocional y mucha gente que se siente sola necesitan compañía y alguien que les brinde cariño." (CNF2)

"Es como una realización personal, porque es algo más... últimamente me da más la impresión de que se trata como de un empleo 'de tercera', pero porque se considera que es una limpieza de casa... hay veces que tienes que sacar tú misma el tiempo para poder cuidar a la persona que te han pedido cuidar." (CNF1)

Estas mujeres son conscientes de que visto desde fuera su trabajo es medible y comparable a las tareas del hogar. Sin embargo, cuidar es "ayudar en muchos sentidos" tantos, que en ocasiones se les exige a las cuidadoras tantas tareas para justificar su salario que han de organizar cómo proporcionar el cuidado:

"Estoy empleando estas horas acompañando a una persona, visto desde fuera parece que sean horas muertas... después de habernos levantado a las 7 de la mañana, en muchas ocasiones de tener relación directa con los médicos, de organizar la compra, la limpieza, de aceptar el mal humor o el estado de ánimo de la persona que cuidas, de hacer su higiene personal, planificar que se va a comer que no..." (CNF1).

Hay una serie de tareas que describen las cuidadoras informales que son invisibles para la sociedad, pero imprescindibles para la vida y el cuidado cotidiano: la presencia, la atención, la preocupación son cualidades que necesita el cuidado para ser un buen cuidado.

Categoría 2: Cuidar como destino. Esta categoría refleja la inexorabilidad del cuidar. Una especie de fatalidad que va ligada a la enfermedad y a la pérdida y que no se escoge, sino que se afronta. En todas las narraciones hay un momento en el que surge la necesidad del cuidado. Bien sea por envejecimiento de los padres, o por la enfermedad del cónyuge, y en esta muestra, mayoritariamente, por la entrada a diálisis.

"Me convertí en cuidadora al ser la persona que convive con el enfermo; además, mi madre se tuvo que venir a vivir con nosotros, para que yo la pudiera atender..." (CF6)

"Yo no escogí cuidar." (CF6)

Aparece con frecuencia el sentimiento de obligatoriedad en el cuidado, de imposición, aunque no se sabe bien de quién o de qué. Convivir con el enfermo facilita el proceso de convertirse en cuidadora.

"Empecé a cuidar más cuando mis padres lo necesitaron." (CF7)

"Yo creo que sí lo escogí, fue una decisión entre todas las hermanas." (CF7).

"Pues empecé a ser cuidadora de verdad cuando nacieron mis hijas, luego también con mis padres ya mayores y ahora, pues apoyando a mi marido con la diálisis peritoneal." (CF5) 
Seco-Lozano et al - Identidad Narrativa en cuidadoras informales familiares y no familiares de personas en diálisis.

El trabajo del cuidado pasa por diferentes etapas: crianza, ancianidad, dependencia o cronicidad, el contexto en el que se desarrolla requiere adaptaciones, pero siempre requiere una acción positiva, un esfuerzo, una inversión de tiempo y de vida.

"Yo no sé si se puede escoger cuidar... creo que eso no se escoge, eso te viene, te toca." (CF5)

Asimismo esta categoría también está ligada a una identidad genuina: la de ser mujer. Las mujeres cuidadoras familiares y no familiares sienten que las cualidades del cuidado son intrínsecas a su manera de sentirse mujer.

"Las mujeres tenemos el gen cuidador" (CNF1)

"Mi madre fue la persona que me gusta imitar, como lo hacía ello intento hacerlo yo" (CF3)

"Mi madre y mi abuela paterna, ellas me cuidaron en la infancia y ya de mayor también." (CF4)

"En la familia, mis padres en general, pero sobre todo mi madre y la madre de mi madre" (CF5)

El cuidado tiene género, el femenino. La identidad del cuidado la adquieren y la trasmiten las mujeres en el ámbito familiar. Ellas ejercen el trabajo del cuidado como un rol asociado a su género y heredado generación tras generación.

Categoría 3: Las familias hacen lo que pueden. Esta categoría también podría titularse: las familias hacen todo lo que pueden, pero en este contexto cada familia sabe qué es lo que puede asumir de este cuidado de larga duración, y la mayoría de las veces el coste es la vida y la salud de la cuidadora familiar que se "sacrifica" en aras de un cuidado absorbente y desgastante sin límite temporal.

Esta responsabilidad asumida desde siempre por las familias las desborda en la cronicidad y en los cuidados de larga duración, donde son necesarios soportes, ayudas para que el cuidado no recaiga en una única persona.

"Lo que necesitamos lo tenemos que pagar: viene un fisioterapeuta a casa y un vecino me ayuda a levantarlo y acostarlo y a la ducha, porque es un peso muerto y yo sola no puedo" (CF3)

"Se cuida con aceptación o con huida" (CF4)

Categoría 4: ¿Quién contará la historia del cuidado? Esta categoría se define por el temor a que el legado de cuidado que estas mujeres realizan diariamente no sea percibido como un valor por la sociedad, ni por sus propias familias, en especial existe un sentimiento de pérdida de la transmisión de su forma de cuidar hacia las generaciones más jóvenes.

"No sé cómo se transmitirá mi manera de cuidar... porque yo no sé si mi hija en un futuro querrá o podrá cuidarme, porque no sé si mi hija me tendrá que llevar a la residencia, pero si yo pudiera escoger quisiera que me cuidaran en mi casa." (CNF2) 
Mayoritariamente la transmisión del cuidado se realiza en la familia, y la figura que prevalece es la materna, pero también surgen otras figuras como los padres, en su función de crianza en la primera infancia y también las abuelas maternas y paternas dejan un legado de cuidado que transciende las generaciones. Ese rol de las abuelas llega a ser muy importante para algunas personas:

"Mi madre y mi abuela paterna, ellas me cuidaron en la infancia y ya de mayor también." (CF4)

"En la familia, mis padres en general, pero sobre todo mi madre y la madre de mi madre" (CF5)

"En mi familia se ha transmitido (el cuidado) porque somos una familia de muchos hermanos: el mayor ha cuidado al más pequeño, la hermana mayor al niñito del vecino... allí todos se cuidaban mutuamente, no era una tarea exclusiva de una persona única de la familia, cualquier pariente te puede cuidar; incluso los vecinos." (CNF2)

Esta transmisión nos habla de una tradición en la que el cuidado es un legado que se hereda en el seno de la familia y especialmente de mujer a mujer. Este legado corre el riesgo de interrumpirse cuando debido a los cambios socio-demográficos, sociales y culturales la mujer se incorpora al mundo laboral y el rol que tradicionalmente ocupaba en el cuidado se transforma.

Categoría 5: Cuidar y Convivir. Esta categoría describe cómo el espacio común donde ocurre el cuidado es el hogar, la intimidad. Este hogar puede tener muchas características: puede tratarse del domicilio de las personas cuidada o del domicilio del cuidador principal. Este entorno de cuidado puede ser familiar exclusivamente o puede convivir la cuidadora no familiar en el domicilio de la persona cuidada.

En esta convivencia, el entorno de cuidado se vehiculiza en varios aspectos: tiempo, presencia, estado de ánimo, disponibilidad, escucha y paciencia. Sin convivir es muy difícil cuidar a personas con grandes necesidades de cuidado. El entorno principal del cuidado es el hogar, y la convivencia es la forma en la que las cuidadoras familiares y no familiares vehiculizan el cuidado. El hogar en el que se cuida es un microentorno, dentro de un macroentorno que se desentiende del cuidado y sus necesidades.

Las narraciones de las mujeres cuidadoras familiares expresan la vivencia de un cuidado en solitario, sin recursos que mitiguen o apoyen la labor cuidadora, en un individualismo de "sálvese quien pueda"

"Pues como se puede: hay personas que pueden cuidar y hay otras que no... cada uno lo lleva como puede." (CF1)

"Yo no he utilizado nada (los recursos), solo mi hija que me ayuda muchísimo... no he utilizado nada... Yo sé que hay alguna ayuda, pero las pides y no sé si te las dan, o no sé si en mi situación que estoy trabajando me la darían... Consigo arreglarme gracias a mi hija que me ayuda físicamente a cuidar a mi padre, si no yo no podría trabajar" (CF1)

Las narraciones de las cuidadoras no familiares aportan la mirada de convertir la relación de cuidado en una relación de familiaridad dentro del espacio íntimo del hogar, lo que conlleva largas horas de disponibilidad y acompañamiento. 
Seco-Lozano et al - Identidad Narrativa en cuidadoras informales familiares y no familiares de personas en diálisis.

"Cuidar a una persona supone un esfuerzo físico pero también va ligado al psíquico porque, aunque esté sentada con la persona estás pendiente; y las horas se pasan muy lentas y tienes que crear una relación y eso es difícil. Eso no es fácil... pero es lo que más satisfacción me da. Lo que más me estresa son las noches cuando las personas están desorientadas o tienen algún problema tipo Alzheimer o demencia. Es muy complicado porque no duermes y no descansas por las noches. Eso a veces las familias no lo entienden, piensan que tú por las noches vas a dormir, pero no. A veces tienes que dormir en la misma habitación incluso que la persona a la que cuidas." (CNF1)

Las cuidadoras no familiares han de saber generar una relación de familiaridad y ejercer el cuidado en el espacio doméstico de las personas cuidadas. La convivencia se convierte en imprescindible para el cuidado.

En esta relación personal que se da en el cuidado las cuidadoras no familiares perciben como algo muy importante la reciprocidad, es decir, sentir algún tipo de soporte o reconocimiento de la persona cuidada, favoreciendo que la convivencia en el cuidado se convierta en una relación de respeto mutuo:

"Pues ha habido de todo (relaciones personales en el cuidado) a veces he pasado como sin pena ni gloria... hay veces que cuesta mucho que te acepten como una persona. Porque realmente te busca la familia (...) y además te busca cuando físicamente no están bien. No es lo mismo que si te busca la persona a la que tienes que cuidar, que eso es muy diferente." (CNF1)

"Cuando alguna vez he atendido a alguien que te ha entrevistado él mismo, porque tenía que cuidarlo a él; es muy diferente la relación a cuando me buscan unos hijos, una familia para cuidar a un familiar. En este caso, la persona ha perdido muchas capacidades: mentalmente no están del todo bien y te tienes que hacer cargo de su higiene y de todo desde el principio. Entonces la aceptación, en algunos casos, cuesta bastante. Porque esa persona no acepta que no esté la familia cuidándole, y no acepta la figura de un cuidador o cuidadora que ha buscado la familia, que paga la familia y que es de fuera de la familia." (CNF1).

En esta cita se pone de manifiesto la necesidad de respetar la autonomía de la persona cuidada. Las personas en diálisis crónica que necesitan del cuidado informal no familiar tienen derecho a escoger la forma en que van a ser cuidadas, y por quién. En muchas ocasiones esta autonomía queda restringida sino eliminada porque es la familia quien decide quién cuidará, cuándo y en qué condiciones, generando conflicto y malestar en la relación persona cuidada-cuidadora informal no familiar.

Categoría 6: El olvido del cuidado. Esta categoría refleja en las narraciones la indiferencia de las generaciones más jóvenes en la participación en el cuidado, pero también en su aprendizaje. El cuidado requiere de un aprendizaje que se lleva a cabo desde la voluntad de imitar una conducta que se admira, que se considera digna, que se percibe valiosa y necesaria. La anterior generación lo ha aprendido sin cuestionarse su valor, dando por hecho que el rol de las mujeres era el cuidado. Ahora que ese rol se ha visto desplazado por otros se desconoce quién las revelará en el cuidado y dudan que sus propias hijas deseen aprender algo acerca de ese cuidado que ya no forma parte de su identidad. 
"Yo transmito mi forma de ver el cuidado a quien me lo pide. Ahora todos saben más que uno... las hijas, los estudiantes, los jóvenes, todos... Creo que los descendientes tienen tanta información que lo que se transmite en casa no tiene interés para ellos, es como si vivieran en dos culturas la de casa y la de ellos... deberían preservarse para las futuras generaciones los valores de respeto, dignidad y humanidad, pero no sé cómo, o si yo lo estoy haciendo." (CF4)

Estas narraciones aportan la incertidumbre de hacia dónde se está desarrollando la tradición del cuidado. Por una parte, las mujeres cuidadoras familiares son conscientes de que las familias, o ellas en solitario no pueden asumir el cuidado de su familiar ante cualquier adversidad. Por otra parte, ven cómo su ejemplo se diluye en la cultura actual y tiene menos peso que nunca. La transmisión que ellas han experimentado parece que en la generación joven no tiene el mismo valor que tuvo para ellas, que se implica (con excepciones) mucho menos en el cuidado. Como ejemplo, en esta muestra sólo hay una nieta implicada en el cuidado de la persona mayor enferma.

Categoría 7: La tarea de acompañar. Esta categoría explora una de las tareas que más simbolizan lo que significa el cuidado informal (ya sea familiar o no familiar), en el entorno de un hogar y en una relación de familiaridad. Acompañar es sin duda la tarea que más tiempo y más dedicación emocional requiere. Es la tarea que menos se valora económicamente y que más necesitan las personas en diálisis.

Acompañar significa en estas narraciones muchas cosas: atender, escuchar, dialogar, dedicar tiempo y atención, ofrecer una parte de ti. En este acompañar se comparten, además actividades diarias como ver la tele juntos, comer, pasear y también se comparten los momentos en los que resurge el dolor crónico, la fatiga, el malestar, el cansancio, la depresión y tristeza de estar en diálisis, la soledad y la pérdida.

En cuanto a las tareas más comunes dentro de las trayectorias de cuidado de gran recorrido encontramos que la higiene corporal y las movilizaciones son dos de las tareas que más desgastan a las cuidadoras informales. A esto se une el deterioro cognitivo de sus seres queridos que les afecta mucho emocionalmente:

"Con mi madre por ejemplo era todo... para todo dependía de mí: darle de comer, la higiene, sacarla de paseo y todo; y eso afectó mucho mi salud. Pero mi padre no tanto; sin embargo, a mí no me afectó tanto mi salud físicamente, sino emocionalmente, simplemente porque yo no aceptaba que mi madre se fue apagando así... yo no aceptaba que mi madre se fuera de la cabeza y que se deteriora tanto y durante tanto tiempo. Eso es lo que te afecta más mentalmente "(CF1)

"Limpieza e higiene, alimentación y apoyo emocional" (CF7)

"Hay muchas cosas que yo no puedo hacer: la higiene, la ducha, levantarlo de la cama... Hemos contratado a una persona que me ayude" (CF3)

De hecho, cuando la dependencia progresa, las cuidadoras familiares no pueden realizar por sí solas la tarea de la higiene y movilización, necesitan la ayuda de otra cuidadora diariamente. 
Seco-Lozano et al - Identidad Narrativa en cuidadoras informales familiares y no familiares de personas en diálisis.

Las tareas de las cuidadoras de recorridos menores, personas autosuficientes en diálisis peritoneal o trasplantada, se centran menos en las necesidades físicas, pero requieren de mucha supervisión: citas médicas, tratamientos, dietas, etc. El tratamiento de diálisis peritoneal requiere una gran implicación en el autocuidado, así como el tratamiento post-trasplante. Esta implicación y adherencia es resultado del apoyo y estímulo de las cuidadoras familiares que planean la alimentación, están pendientes de la evolución, del control y seguimiento del tratamiento:

"Pues en estos momentos hemos descansado mucho con el trasplante. Hemos sentido un gran alivio... pero hemos pasado mucho estrés, mucha preocupación. Primero empezamos en peritoneal, tuvo una peritonitis, hubo que quitarle el catéter; luego empezamos con la hemodiálisis y hasta el trasplante lo hemos pasado muy mal" (CF6)

Las trayectorias del cuidado de las personas en diálisis son largas y tortuosas, con obstáculos de índole muy diversa: físicos, emocionales, sociales...Todas estas situaciones críticas, y la vivencia de la incertidumbre son elementos estresantes para las cuidadoras informales familiares.

"Control de constantes, alimentación, acompañamiento, confort" (CF4)

"Seguimiento de la dieta, supervisión del tratamiento, acompañar a revisiones del trasplante" (CF6)

"Las tareas que hago son variadas, la atiendo en lo que necesita: a veces le preparo la ducha y ella prefiere ducharse sola, pero yo estoy pendiente" (CF2)

La amplia variedad de tareas que requiere el trabajo de cuidado implican tiempo, atención, supervisión y dedicación completa.

"De momento, él es autosuficiente, yo sólo lo acompaño a veces a alguna visita, pero sobre todo, es apoyo emocional" (CF5)

Las tareas que más esfuerzo psíquico requieren tienen que ver con aspectos emocionales: por ejemplo, cuando la persona cuidada transgrede la dieta o el tratamiento. 0 simplemente cuando el estado de ánimo de la persona cuidada está muy afectado y negativo.

Por otra parte, el miedo a eventos adversos que empeoren la situación también es causa sobrecarga:

"Es el miedo a que se olvide algo o suceda algo inesperado" (CF2)

"Cumplimiento del tratamiento" (CF4)

"Cuando se pone nervioso" (CF3)

La adherencia al tratamiento de las personas en diálisis es imprescindible y es un gran problema en las unidades de diálisis. Incluye la toma de medicación oral (suele oscilar entre 8 y 12 tipos de medicación diferente a diferentes horas del día y administradas de diferentes formas) junto a la restricción hídrica, de sodio, de potasio y de fósforo. Si a esto le unimos que tengan otra enfermedad crónica como diabetes mellitus, las necesidades de tratamiento y control de dieta son aún mayores. No es nada fácil cocinar 
alimentos y planificar una dieta para un enfermo renal, requieren de unas cocciones especiales y abundan los alimentos "prohibidos" por sus altos contenidos en potasio, sodio o fósforo.

Las tareas que sobrepasan sus capacidades de cuidado tienen mucho que ver con las complicaciones que puedan surgir y con la imposibilidad física de su movilización:

"Pensar si sangra la fístula en casa, porque toma Sintrón $\gtrless$ y ya le ha pasado, y no sabes qué hacer en plena noche con tanta sangre, y él que se pone muy nervioso, o que se mareé y que pierda la cabeza" (CF3)

"En este momento me sobrepasan las tareas físicas porque no se puede movilizar en absoluto" (CF7)

Las tareas que más satisfacción les producen son todas aquellas que suponen un alivio, una mejora, un pequeño avance o bienestar en sus seres queridos.

"Cuando se ven resultados positivos" (CF4)

"Cuando veo que está bien" (CF3)

Las tareas de apoyo al autocuidado son una gran fuente de satisfacción para las cuidadoras familiares. Recordemos que para las personas en diálisis el autocuidado era muy importante y las hacía sentirse muy bien:

"Pues cuando he visto que él ha podido cuidarse por sí mismo y que no me deja que le ayude, que lo hace todo y que está más contento" (CF5)

"Ver como ella se implica y se esfuerza por hacer las cosas por sí misma" (CF2)

El recorrido de las cuidadoras familiares de personas trasplantadas ha tenido un desenlace muy satisfactorio, los momentos angustiantes quedan atrás, y se vive como un premio, una lotería, un milagro:

"Pues ahora que ya está trasplantado miras hacia atrás y piensas en todo lo que has pasado, y cómo ha sido posible que hayas vivido momentos tan tensos, tan angustiosos, que no sabía si vas a salir... y ahora al verlo recuperado parece que tenga una vida nueva" (CF6)

Para las cuidadoras no familiares las tareas que más les cuestan físicamente, y las que más les sobrecargan son muy parecidas a las cuidadoras familiares: movilización, higiene y el deterioro cognitivo son tres aspectos que dificultan mucho el cuidado:

"Hay que estar muy pendientes de ellas por las noches porque pierden la orientación. He tenido personas que por las noches pues desvariaban o se orinaban encima. Momentos con muchas crisis, que lo cuentas a la familia y no te creen; y hay veces que la familia tiene que vivir esa situación para que te crean y entiendan que el cuidado cuando hay agresividad o algún tipo de problema mental es muy difícil" (CNF1)

Y no sólo se requiere atención y vigilancia, sino una actitud empática de forma casi permanente que es difícil a veces de conseguir: 
Seco-Lozano et al - Identidad Narrativa en cuidadoras informales familiares y no familiares de personas en diálisis.

"Estar al cuidado de una persona mayor; siempre con la sonrisa en la boca, te cuenta una anécdota 40. 000 veces y tú la tienes que aceptar y acabas un poco en un bucle... como que te aburre el tema que te está contando, pero claro tienes que estar ahí... ves que esa persona su mente no rige como una persona normal. Tienes que estar ahí aguantando con mucha paciencia y también cariño. Hay veces que se ponen violentos... salvar esa situación sin hacerle daño, pero también protegiéndote es sacrificado también es complicado llegar a un punto de equilibrio y cuando tienes que levantarlo de la cama si no lo haces de la manera adecuada le haremos daño y nos dañaremos la espalda" (CNF2)

También lo que más sobrepasa a las cuidadoras no familiares son los eventos adversos que puedan ocurrir junto a la exigencia de un trabajo de cuidado total: tareas domésticas, unido al cuidado del familiar las 24 horas del día. Los horarios de 24 horas sobrepasan a las cuidadoras no familiares:

"Cuando me ha tocado hacer noche con las personas que he cuidado y he pensado: 'Madre mía y si pasa algo... y si se mueren aquí, qué hago, a quién llamo, cómo me comporto?'. Hay muchas veces que tienes miedo que te echen... entonces muchas veces sobrepasa mi capacidad de cuidado. Pero te dicen: 'Mira que la fulanita esta me lava, me plancha, me limpia la casa, hace a la abuela, la saca a pasear...'. Y en ese sentido estamos sobrepasadas. Vale que necesito el dinero, pero no lo puedo hacer todo y en ese sentido me siento sangrada" (CNF2)

El cuidado es percibido por la sociedad como un tiempo perdido, devaluado. Las cuidadoras informales no familiares sienten como el trabajo de cuidado que Ilevan a cabo es poco valorado.

Categoría 8: Confianza y cariño: cualidades del cuidado. En esta categoría se visibilizan las cualidades que hacen falta en una relación de cuidado. Para poder cuidar a una persona en su casa y cerca de su familia es imprescindible que el cuidado tenga dos cualidades: confianza y cariño. Esta confianza y este cariño serán las bases para establecer una relación de familiaridad en la que el cuidado pueda darse con respeto, dignidad y empatía. A la vez este cariño y esta confianza han de preservar el respeto y la dignidad de la persona que cuida. Promover la confianza es difícil, y propiciar el cariño lo es más aún. Se trata de asumir esas cualidades con responsabilidad, con madurez. Todo el mundo necesitará ser cuidado algún día, pero no todo el mundo va a ser capaz de desarrollar las habilidades y cualidades que requiere el buen cuidado de:

"Cariño, comprensión, existencia de reciprocidad, preocupación." (CF2).

"Cuidado es obligación, cansancio, te quema." (CF3)

"Es amor, compromiso, compasión y obligación." (CF4)

"El cuidado, pues.... es una responsabilidad muy grande. A veces satisfacción, y a veces, una carga." (CF5)

Las interacciones negativas entre cuidadoras familiares y no familiares en esta muestra han surgido por conductas de abandono, descuido y estafa por parte de cuidadoras no familiares. Y por otro lado, la insensibilidad y discriminación por la edad de parte de algún familiar. Estas interacciones negativas muestran que son imprescindibles unas habilidades/cualidades para un buen cuidado, y que el cuidado informal necesita 
urgentemente acuerdos y orientación para definir lo que se entiende por un buen cuidado. También en esta categoría se profundiza en la propia relación de cuidado, qué calidad tiene esta relación para las cuidadoras, qué les aporta...

"La relación que tengo es que son muy cercanas a mi familia (...). Mi madre no podía aportarme nada... pobrecita, porque tenía el deterioro cognitivo, pero mi padre sí, cariño, alegría..." (CF1)

"Me apoya muchísimo (la persona que cuida a su madre)" (CF2)

"La relación que tengo es marital y aunque tengo personas en las que puedo delegar el cuidado... pero no quiero implicar a los hijos mientras pueda (... él (marido) raramente me puede ofrecer soporte emocional porque somos muy opuestos" (CF4)

En estas citas el cuidado vuelve a emerger como un soporte emocional, más que como una acción en sí misma. Cuidar también es preocuparse, escuchar, apoyar, acompañar, comprender...

"La relación que tengo (con mi marido) es muy buena, claro que a mí también me apoya" (CF5)

Se trata de relaciones muy cercanas y especiales y existe reciprocidad, es decir la persona cuidada apoya, acompaña, ofrece cariño y atención emocional a la persona que cuida. Si bien es cierto que mayoritariamente son buenas relaciones, también surge en la muestra relaciones no tan fluidas o armónicas, pero en las cuales también hay un compromiso de cuidado.

Las cuidadoras familiares que pueden delegar en alguien (hija o hermanas) son pocas (CF1 y CF7). El resto de cuidadoras familiares cuida en solitario.

Sólo las cuidadoras CF6 y CF3, que son cuidadoras de gran recorrido con muchos años a sus espaldas, conocen a la asociación Alcer Turia, una asociación formada por enfermos renales que sirve de apoyo a ellos y a sus familias. Las demás cuidadoras no se han puesto en contacto nunca con ninguna asociación de ayuda para el cuidado.

Temática análisis hermenéutico. El contexto en el que vivencian el cuidado las cuidadoras informales de las personas en diálisis tiene varias dimensiones. Realizan un trabajo de cuidado en un contexto social donde el cuidado no es un valor, donde tradicionalmente ha sido llevado a cabo por las mujeres naturalizado por su género.

En este contexto de cuidado aparecen tres temas de significado relevante en esta muestra. El primero aborda la relación acerca de lo que significa vivir para cuidar. Esta experiencia es común a las cuidadoras tanto familiares como no familiares. El segundo tema explora la balanza entre lo que se pierde y lo que se gana cuidando, como una expresión de valores que apoyan el cuidado como un bien. El tercer tema aborda con dificultad las tensiones en el cuidado informal entre cuidadora familiar y no familiar, tensiones que surgen de un vacío que regule las relaciones laborales y también de la necesidad urgente de una orientación básica acerca de lo que significa un buen cuidado. 
Seco-Lozano et al - Identidad Narrativa en cuidadoras informales familiares y no familiares de personas en diálisis.

Tema 1: Vivir para cuidar. Las cuidadoras familiares y no familiares viven cuidando. Porque cuidar para ellas no es sólo dedicar un tiempo o realizar unas actividades. Implica también una actitud y una ocupación mental, en muchas ocasiones, una preocupación de la que no es fácil evadirse. Porque quien cuida lo hace desde su vida, desde su afecto, y no delimita franjas horarias, por lo que cuando la necesidad de cuidado emerge sólo queda cuidar, que es decir que sólo queda vivir, vivir cuidando.

Hasta ahora este "vivir para cuidar" tenía herederas que no podían o no querían escapar del legado de deuda y obligación familiar que habían contraído al ser mujeres, y al sentirse ligadas e interpeladas por el afecto, mayoritariamente respondían valientemente a la llamada del cuidado.

Pero estas herederas del cuidado han criado hijas que están construyéndose una identidad menos condicionada, y el cuidado que durante generaciones ha estado asegurado, necesita un espacio común para ser compartido y vivido, tal vez incluso una sociedad nueva.

Tema 2: Perder cuidando y ganar perdiendo. Con este tema se profundiza en el misterio que significa para las cuidadoras familiares y no familiares la forma en que su entrega, dedicación y en muchas ocasiones sacrificio revierte en forma de realización, satisfacción personal, crecimiento. De alguna manera este pensamiento de que están "ganando" les compensa, y es que claro que ganan, ganan una identidad que demuestra el valor del cariño, la paciencia, la empatía.

Pero hay muchas ocasiones, en que llevan tanto tiempo cuidando en solitario que es muy difícil sentir la ganancia, porque en ese cuidado están perdiendo la salud, la alegría, la tranquilidad: cuando las cuidadoras familiares de las personas en diálisis sienten que ya no pueden más, cuando su trayectoria ha sido larga, dura, amarga.

Tema 3: Tensiones en el cuidado informal. Existen tensiones evidentes en el cuidado informal. Por una parte, cuando aparece la enfermedad y su cronificación, la cuidadora familiar siente que no tiene elección, que "le toca". A partir de aquí, algunas cuidadoras familiares sienten que no pueden escoger, aunque otras realizan una elección consciente de lo que implica responsabilizarse del cuidado. Otro de los momentos de tensión es cuando la familia decide buscar ayuda de una cuidadora informal no familiar: la adaptación al contexto familiar de cuidado, la necesidad de confianza mutua, la relación económica o laboral, son fuente de tensiones que han de irse resolviendo.

Estas tensiones se viven en ambas direcciones: es mucho lo que se espera de una cuidadora informal no familiar: tareas y actividades exigentes en horarios muy amplios, además de cualidades como la paciencia, la empatía, la honradez. Las cuidadoras no familiares no suelen pertenecer a ningún tipo de colectivo que defienda sus derechos, o que regule sus condiciones laborales. Tal vez como han llegado al trabajo de cuidado como alternativas a sus distintas formaciones, ni siquiera ellas mismas lo ven como un trabajo profesionalizado. En esta muestra sólo una cuidadora no familiar pertenecía a un Servicio de Atención a la Dependencia. Solo dos de ellas habían recibido cursos y formación sobre el cuidado. 
A esto se une que en muchas ocasiones la persona cuidada no acepta la presencia de una cuidadora no familiar, que ni siquiera ha sido escogida por ella. El propio acuerdo al que Ilega la familia y la cuidadora informal es un contexto que propiciará el cuidado o se convertirá en una barrera para la confianza y la relación de familiaridad.

Otro foco de tensiones es consensuar las condiciones laborales, los horarios, retribuciones, días libres, condiciones en que se va a llevar a cabo la convivencia etc. Como también lo es contrastar el grado de profesionalización de las cuidadoras no familiares. Sin convenios, sin sindicatos sin legislación que regule el trabajo del cuidado en los hogares estas relaciones entre las cuidadoras familiares y las no familiares son propensas a los malentendidos, la desinformación, los abusos o la desconfianza e incluso el engaño.

\section{DISCUSIÓN}

Mc Donald et al., (2017) utilizan también la teoría fundamentada en un estudio acerca de las necesidades de aprendizaje de los cuidadores informales en dolencias crónicas. Sus resultados subrayan la gran necesidad de soporte por parte de los profesionales sanitarios que requieren los cuidadores informales en la cronicidad. Si bien su trabajo no valora la vivencia del cuidado de los cuidadores sólo su necesidad de formación.

Por otra parte, Moreno et al., (2016) describen cómo las cuidadoras en cuidados de larga duración interiorizan el rol de cuidadoras hasta el punto de formar parte integrante de su personalidad. Profundiza en la preferencia de un cuidado en casa, como contraposición a un cuidado institucionalizado. Cuando debido a los cambios sociodemográficos actuales, como la incorporación de la mujer en el ámbito laboral y la reducción de la familia extensa, el cuidado informal no puede llevarse a cabo de la forma tradicional en la que se realizaba, es decir, por la madre, hija, hermana o familiar de la persona necesitada de cuidado, pasa a ser delegado en otra cuidadora informal, también mujer, a la que se transfieren estas tareas de cuidado a cambio de algún tipo de remuneración. Estos resultados respaldan los resultados obtenidos en esta investigación, si bien nuestro estudio complementa el conocimiento acerca del cuidado informal describiendo sus cualidades más positivas, las tareas más invisibles, como la de acompañar y las tensiones que acompañan la relación entre cuidadoras familiares y no familiares.

Por otro lado, las experiencias de las cuidadoras informales familiares de personas que reciben diálisis reflejan un alto nivel de sobrecarga, se sienten aislados socialmente y sobrepasados por el cuidado, necesitando incluso más ayuda y atención para su propia salud, lo hemos visto en esta investigación y lo corroboran diferentes autores. La naturaleza compleja de los cuidados que requieren las personas en diálisis hace que necesiten ayuda para elaborar formas de resiliencia que les puedan dar soporte a través del tiempo y de los años de cuidado (Hoang, Green y Bonner, 2018).

\section{Conclusiones}

En primer lugar, existe un vacío de acuerdos y de arbitrajes que generan tensiones, abusos y malas prácticas entre el cuidado familiar y el cuidado no familiar. No se sabe quién ha de intermediar en estas relaciones. También existe un desconocimiento acerca de cuáles son las tareas del cuidado informal, siendo algunas tan fundamentales como: 
el acompañamiento, la escucha, la supervisión y organización de actos de cuidado totalmente invisibles para la sociedad.

En segundo lugar la identidad narrativa del cuidado surge del sentimiento en las cuidadoras familiares del "cuidado como destino", y en las cuidadoras no familiares como la dotación intrínseca de "su forma de ser mujer", que también se percibe como una especie de fatalismo o de inexorabilidad. Ante el impacto de la enfermedad, las cuidadoras familiares asumen el cuidado de la mejor forma que pueden, y en muchas ocasiones, sin otro apoyo familiar. Por su parte, las cuidadoras no familiares inmigrantes acceden al cuidado como una vía para cambiar de vida en su proceso de emigrar a España.

Cuando el cuidado a su familiar ha minado la salud de las cuidadoras, el ocio, las relaciones sociales y el estado de ánimo, no sólo están perdiendo ellas, sino sus familias, el entorno, la sociedad que permite, con indiferencia, que circunstancias desfavorables, como la enfermedad renal crónica terminal, arrastre a las personas en diálisis y a sus cuidadoras a una vida sin esperanza. En estas ocasiones, quien pierde es la sociedad en su conjunto, y quien ya ha ganado, porque siempre al dar se gana, son las cuidadoras que han apostado su vida al cuidado

Al mismo tiempo, aunque las instituciones asumen el cuidado más tecnificado de las personas en diálisis, la cronicidad es una característica que prolonga indefinidamente en el tiempo la necesidad de cuidado de estas personas. Esta cronicidad derivará con el paso de los años, en una dependencia que cada vez requerirá más trabajo de cuidado informal, siendo este cuidado informal imprescindible para las personas en diálisis crónica.

En tercer lugar, la relación de cuidado no puede construirse sin dos ingredientes fundamentales: el cariño y la confianza. Estas cualidades son vinculantes y recíprocas en el cuidado informal en el contexto de una relación de familiaridad. Cuidar es convivir y la vida de las cuidadoras informales de las personas en diálisis crónica es, en muchas ocasiones, vivir cuidando.

\section{REFERENCIAS}

Brassolotto J. y Daly T. (2016). Domesticating dialysis: A feminist political economy analysis of informal renal care in rural British Columbia. Can Geogr, 60(4): 519-529. doi: 10.1111/cag.12319. Epub 2016 Oct 6. Recuperado de: https://www.ncbi.nlm. nih.gov/pubmed/29307896

Comars D'Argemir, D. (2015). Los cuidados de larga duración y el cuarto pilar del sistema de bienestar. Revista de Antropología Social, 14, 374-404. http://dx.doi. org./10.5209/rev_RASO.2015,24,50663.

Domingo, T. (2007). Bioética y hermenéutica. La aportación de Paul Ricoeur a la Bioética. Veritas II, 17, 281-312.

Durán, M.A. (2018). La riqueza invisible del cuidado. Valencia. España: Universidad de Valencia. 
Hoang, VL., Green, T., y Bonner, A. (2018). Informal caregivers' experiences of caring for people receiving dialysis: A mixed-methods systematic review. Journal of Renal Care, 44 (2): 82-95. doi: 10.1111/jorc. 12235.

Iacub, R. (2013). El envejecimiento desde la identidad narrativa. Revista Argentina de Psiquiatria, XXI: 298-305. Recuperado de: http://pdfhumanidades.com/sites/ default/files/apuntes/

McDonald J, McKinlay E, Keeling S, Levack W. The 'wayfinding' experience of family carers who learn to manage technical health procedures at home: a grounded theory study. Scand J Caring Sci. 2017 Dec;31(4):850-858. doi: 10.1111/scs.12406. Epub 2017 Jan 25

More, P. (2018). Los límites del cuidado. Organización de la asistencia a domicilio para personas mayores en Madrid y París. Revista Internacional de Sociología, 76 (1): e086. https://doi. org/10.3989/ris.2018.76.1.16.25

Moreno, S., Recio, C., Borrás, V., y Torns, T. (2016). Significados e Imaginarios de los cuidados de larga duración en España. Una aproximación cualitativa desde los discursos de cuidadoras. International Journal on Collective Identity Research, 1 (145): 1695-1694. http:/dx.doi.org/10.1387/pcesic.15195.

OHare, AM, Szarka, J. McFraland, LV., Vig, EK, Sudore, RL., Crowley, S., Reinke, LF, Triverdi, R., y Taylor, JS. (2018). Emotional impact of Illness and Care on Patientes with Advanced Kidney Disease. Clinical Journal American Nephrology, 13(7): 10221029. doi:10.2215/CJN.14261217.

Parra, O. (2014). La enfermedad cuenta. Narrativa y Bioética del cuidado. Revista Latinoamericana de Bioética, 14 (1): 48-61. Recuperado de: http://www.redalyc. org/pdf/1270/127031381005.pdf

Ramos del Río, B., y Jarillo, E. C. (2014). El cuidado informal de personas con enfermedad renal crónica. Una mirada desde la salud colectiva a la teoría de las representaciones sociales. Enclaves del Pensamiento, VIII (15): 195-216.

Rogero J. (2009) Los tiempos del cuidado. IMSERSO-Premio Infanta Cristina. Colección Estudios. Serie Dependencia no 120011. Edita Ministerio de Sanidad y Política Social. Madrid.

Romero E., Mac Causland, Y. y Solórzano E. (2014). El cuidador familiar del paciente renal y su calidad de vida. Salud Uninorte, 30 (2): 146-157. Recuperado de: http:// dx.doi.org/10.14482/sun.30.2.2185

Ricoeur, P. (1996). Si mismo como otro. Editorial Siglo Veintiuno, S.A. Madrid, España.

Ricoeur, P. (1999) Historia y Narratividad. Editorial Paidós. Madrid, España.

Zamarripa E., Tamez B. y Ribeiro, M. (2017). Repercusiones del cuidado informal en la vida laboral y personal de las mujeres cuidadoras. Revista Internacional de Trabajo Social y Bienestar, 6: 47-56. 